\title{
BLOW-UP OF SMOOTH SOLUTIONS TO THE NAVIER-STOKES EQUATIONS FOR COMPRESSIBLE ISOTHERMAL FLUIDS*
}

\author{
DAPENG DU ${ }^{\dagger}$, JINGYU LI ${ }^{\ddagger}$, AND KAIJUN ZHANG§
}

\begin{abstract}
It is shown that the one-dimensional or two-dimensional radially symmetric isothermal compressible Navier-Stokes system has no non-trivial global smooth solutions if the initial density is compactly supported.
\end{abstract}

Key words. Compressible Navier-Stokes equations, isothermal fluids, blow-up.

AMS subject classifications. 76N10, 35Q30, 35B44.

\section{Introduction}

In this paper we consider the blow-up problem for the Navier-Stokes equations of compressible isothermal fluids,

$$
\left\{\begin{array}{l}
\partial_{t} \rho+\operatorname{div}(\rho u)=0, \\
\partial_{t}(\rho u)+\operatorname{div}(\rho u \otimes u)-\mu \Delta u-(\lambda+\mu) \nabla \operatorname{div} u+\nabla P(\rho)=0,
\end{array}\right.
$$

with initial data

$$
\rho(x, 0)=\rho_{0}(x), \quad u(x, 0)=u_{0}(x), \quad x \in \mathbb{R}^{n}, \quad 1 \leq n \leq 2 .
$$

This system describes the motion of viscid gas. $u \in \mathbb{R}^{n}, \rho, \mu$, and $\lambda$ denote the velocity, the density, the coefficient of viscosity and the second coefficient of viscosity, respectively. The pressure $P(\rho)$ satisfies

$$
P(\rho)=a \rho
$$

for a positive constant $a$.

Mathematically, system (1.1) is a quasilinear transport-parabolic system. There are many important questions regarding this system. One of them is whether the solutions will develop singularities from smooth initial data. Xin [8] first proved that when the initial densities are compactly supported, any smooth solutions to the full compressible Navier-Stokes equations in the absence of heat conduction will blow up in finite time for any spatial dimension, and this feature also holds for the isentropic flows (i.e. $P(\rho)=a \rho^{\gamma}$ with $\gamma>1$ ) in one dimensional case. Cho and Jin [1]

\footnotetext{
*Received: May 4, 2012; accepted (in revised form): July 14, 2012. Communicated by Yaguang Wang.

Du's work is partially supported by the Chinese NSF (No. 11001043) and the Chinese Postdoctoral Science Foundation (No. 20090460074); Li's work is partially supported by the Chinese NSF (No. 11101073), the Fundamental Research Funds for the Central Universities (No. 10QNJJ001), and the China Postdoctoral Science Foundation funded project; Zhang's work is partially supported by the Chinese NSF (No. 11071034) and the Fundamental Research Funds for the Central Universities (No. 111065201).

${ }^{\dagger}$ School of Mathematics and Statistics, Northeast Normal University, Changchun 130024, P.R. China (dudp954@nenu.edu.cn).

${ }^{\ddagger}$ Center for Partial Differential Equations, East China Normal University, 500 Dongchuan Road, Shanghai, 200241, P.R. China, and School of Mathematics and Statistics, Northeast Normal University, Changchun 130024, P.R. China (lijy645@nenu.edu.cn).

$\S$ School of Mathematics and Statistics, Northeast Normal University, Changchun 130024, P.R. China (zhangkj201@nenu.edu.cn).
} 
extended Xin's work [8] to the case of fluids with positive heat conduction. Under an additional assumption that one of the components of initial momentum is not zero, Tan and Wang [7] gave a much simpler proof of the result of Cho and Jin [1]. If the initial data do not have compact support but rapidly decrease, for $n \geq 3$ and $\gamma \geq \frac{2 n}{n+2}$, Rozanova [5] proved that any smooth solutions to the full compressible Navier-Stokes equations with positive heat conduction still blow up in finite time. Very recently, for the full compressible Navier-Stokes equations without heat conduction, Xin and Yan [9] extended the blow-up result to the case that the initial density is isolated, i.e. for some open set $U$, supp $\rho_{0} \cap U$ is compact in $U$. It should be noted that Makino, Ukai, and Kawashima [4] were the first to prove the blowup of smooth solutions to a three dimensional compressible Euler system with compact initial density and velocity.

It is worth mentioning that very recently, Huang, Li, Luo, and Xin [2] proved that any smooth radially symmetric solutions to the two-dimensional isentropic fluids with compactly supported initial densities blow up. And they also proved in [3] a very interesting result - that the three-dimensional isentropic compressible Navier-Stokes equations with compactly supported initial densities are globally well-posed for small initial data with some compatibility condition.

Besides the full ones and the isentropic ones, the isothermal compressible NavierStokes equations are also of great physical significance. From the viewpoint of mathematical structures, the isothermal case could be looked as the endpoint of the isentropic case. Roughly speaking, we show that if the initial data are nontrivial and radially symmetric, and the initial densities are compactly supported, then the smooth solutions to (1.1) will blow up in finite time. More precisely, we prove

Theorem 1.1. Assume that

$$
\mu>0, \quad \lambda+\frac{2}{n} \mu>0,
$$

and that $\rho_{0}(x)$ has compact support. Suppose that one of the following conditions holds:

(i) $n=1$;

(ii) $n=2$, and the initial data are spherically symmetric, i.e.

$$
\rho=\rho(|x|, t), \quad u=\frac{x}{|x|} \bar{u}(|x|, t) .
$$

Then the solution $(\rho, u) \in C^{1}\left([0, T], H^{m}\left(\mathbb{R}^{n}\right)\right)(m>2)$ to system (1.1) with nontrivial initial density will blow up in finite time.

REMARK 1.1. Our method also works for the isentropic case and full case if the initial density is compactly supported. The proofs are essentially are the same. One reason is that all these systems have similar energy conservation and the same momentum equation.

One key ingredient in the existing proof for the isentropic case and full case is a differential inequality on some functional $I_{\gamma}(t)$. More precisely, in [8] (see the proof of Proposition 2.1 in [8]) the functional is

$$
I_{\gamma}(t)=\left\{\begin{array}{lr}
\int_{\mathbb{R}^{n}}|x-u(x, t)(t+1)|^{2} \rho(x, t) d x+\frac{2(t+1)^{2}}{\gamma-1} \int_{\mathbb{R}^{n}} p(x, t) d x, & 1<\gamma<1+\frac{2}{n}, \\
\int_{\mathbb{R}^{n}}|x-u(x, t) t|^{2} \rho(x, t) d x+\frac{2 t^{2}}{\gamma-1} \int_{\mathbb{R}^{n}} p(x, t) d x, & 1+\frac{2}{n} \leq \gamma<+\infty,
\end{array}\right.
$$


and the differential inequality is

$$
\frac{d}{d t} I_{\gamma}(t) \leq \frac{2-n(\gamma-1)}{t+1} I_{\gamma}(t)
$$

This inequality works when $\gamma$ is bigger than 1 . At this stage, it seems unclear how to extend the methods in the full and isentropic cases to the isothermal one.

To overcome the difficulty above, we choose another functional $\int_{\mathbb{R}^{n}} \rho u \cdot x d x$. The proof roughly goes as follows. First we show that the density keeps being compactly supported, which is similar to that of [8]. This idea was also used in [6] for a system of conservation laws and a nonlinear wave equation. Then we integrate the momentum equation by the weight $x$, which gives an integral identity. It consists of three terms. One important observation is that, as time grows, two terms keep bounded, but one term grows linearly. This fact implies no nontrivial global smooth solution exists, otherwise, there is a contradiction. The details will be presented in Section 2 .

\section{Proof of Theorem 1.1}

In this section we give the proof of Theorem 1.1, which consists of four steps.

Step 1. The density $\rho$ is compactly supported all the time.

Because the initial density $\rho_{0}(x)$ has compact support, there exists a constant $R>0$ such that

$$
\operatorname{supp} \rho_{0}(x) \subseteq B_{R} .
$$

Denote by $x(t ; \bar{x})$ the particle path starting from $\bar{x}$, i.e. $x(t ; \bar{x})$ satisfies the following equation:

$$
\left\{\begin{array}{l}
\frac{d x}{d t}=u(x, t), \\
x(0 ; \bar{x})=\bar{x},
\end{array}\right.
$$

where $u$ is the velocity. Denote by $\Omega(t)$ the closed region that is the image of $B_{R}$ under the flow map $(2.2)$,

$$
\Omega(t):=\left\{(x, t) \mid x=x(t ; \bar{x}), \bar{x} \in B_{R}\right\} .
$$

Note that on the particle path $x=x(t ; \bar{x})$, the density $\rho$ satisfies a homogeneous ordinary differential equation. Thus, if $\rho$ is zero at some initial position $\bar{x}$, then on the particle path starting from $\bar{x}, \rho$ will be zero all the time. By (2.1), we see that

$$
\operatorname{supp}_{x} \rho(x, t) \subseteq \Omega(t) .
$$

Subsequently, using the momentum equation of (1.1), we get

$$
\mu \Delta u+(\lambda+\mu) \nabla \operatorname{div} u=0 \quad \text { on }\{t\} \times \mathbb{R}^{n} \backslash \Omega(t) .
$$

In the one-dimensional case, owing to (1.4), we deduce from (2.3) that

$$
u_{x x}=0 \quad \text { on }\{t\} \times \mathbb{R} \backslash \Omega(t) .
$$

This in combination with the condition $u(\cdot, t) \in H^{m}\left(\mathbb{R}^{1}\right)$ implies that

$$
u(x, t) \equiv 0 \quad \text { on }\{t\} \times \mathbb{R} \backslash \Omega(t) .
$$


Therefore, in view of (2.2), we derive

$$
\Omega(t)=\{t\} \times \Omega(0) \subseteq\{t\} \times B_{R}, \quad \forall 0<t<T .
$$

In the two-dimensional case, because $u(x, t)=\frac{x}{|x|} \bar{u}(|x|, t)$ for some radially symmetric function $\bar{u}$, we have from (2.3) that

$$
\bar{u}_{r r}+\left(\frac{\bar{u}}{r}\right)_{r}=0 \quad \text { on }\{t\} \times \mathbb{R}^{2} \backslash \Omega(t) .
$$

Here $r:=|x|$. Using the condition $u(\cdot, t) \in H^{m}\left(\mathbb{R}^{2}\right)$ again, it follows that

$$
\bar{u}_{r}+\frac{\bar{u}}{r}=0 \quad \text { on }\{t\} \times \mathbb{R}^{2} \backslash \Omega(t) .
$$

One can easily compute that the general solution to this equation is

$$
\bar{u}(r, t)=\frac{C(t)}{r},
$$

where $C(t)$ is a constant dependent on $t$. Because $u \in C\left([0, T], L^{2}\left(\mathbb{R}^{2}\right)\right)$, we get for any $t \in[0, T]$,

$$
\int_{\omega_{2}} \int_{0}^{+\infty}|\bar{u}(r, t)|^{2} r d r d S<+\infty
$$

By (2.4),

$$
C^{2}(t) \int_{\omega_{2}} \int_{0}^{+\infty} r^{-1} d r d S<+\infty,
$$

which implies $C(t)=0$ for any $t \in[0, T]$. Then

$$
\bar{u}(r, t) \equiv 0 \quad \text { on }\{t\} \times \mathbb{R}^{2} \backslash \Omega(t) .
$$

Therefore, $\Omega(t)=\{t\} \times \Omega(0) \subseteq\{t\} \times B_{R}$.

Step 2. Averaging the equation by a weight.

We multiply the momentum equation of (1.1) by weight $x$ and integrate the resulting equation on the whole space to derive

$$
\int_{\mathbb{R}^{n}} \rho u \cdot x d x-\int_{\mathbb{R}^{n}} \rho_{0} u_{0} \cdot x d x=\int_{0}^{t} \int_{\mathbb{R}^{n}}\left(\rho|u|^{2}+n a \rho\right) d x d \tau,
$$

where we have used the isothermal condition (1.3). In view of the fact that

$$
\int_{\mathbb{R}^{n}} \rho(x, t) d x=\int_{\mathbb{R}^{n}} \rho_{0}(x) d x:=m_{0},
$$

and that $\operatorname{supp}_{x} \rho(x, t) \subseteq B_{R}$, we then get

$$
\int_{B_{R}} \rho u \cdot x d x-\int_{B_{R}} \rho_{0} u_{0} \cdot x d x=\int_{0}^{t} \int_{\mathbb{R}^{n}} \rho|u|^{2} d x d \tau+\text { nam }_{0} t .
$$

Obviously, the right-hand side of (2.7) grows linearly in $t$. Next we show that the left-hand side of (2.7) is bounded. This will give the contradiction with the induction 
hypothesis that the Navier-Stokes system has a global solution. To get the boundedness of the left-hand side of (2.7), we need to prove energy conservation.

Step 3. Energy conservation.

Multiplying the density equation of (1.1) by $\ln (\rho+\epsilon)$, where $\epsilon>0$ is a small constant, we get

$$
\int_{\mathbb{R}^{n}} \rho_{t} \ln (\rho+\epsilon) d x=-\int_{\mathbb{R}^{n}} \operatorname{div}(\rho u) \ln (\rho+\epsilon) d x .
$$

It is easy to see that

$$
\begin{aligned}
\rho_{t} \ln (\rho+\epsilon) & =[\rho \ln (\rho+\epsilon)]_{t}-\rho[\ln (\rho+\epsilon)]_{t}=[\rho \ln (\rho+\epsilon)]_{t}-\frac{\rho \rho_{t}}{\rho+\epsilon} \\
& =[\rho \ln (\rho+\epsilon)]_{t}-\rho_{t}+\epsilon[\ln (\rho+\epsilon)]_{t},
\end{aligned}
$$

and that

$$
\begin{aligned}
\int_{\mathbb{R}^{n}} \operatorname{div}(\rho u) \ln (\rho+\epsilon) d x & =-\int_{\mathbb{R}^{n}} \frac{\rho u \cdot \nabla \rho}{\rho+\epsilon} \\
& =-\int_{\mathbb{R}^{n}} u \cdot \nabla \rho d x+\int_{\mathbb{R}^{n}} u \cdot \nabla[\epsilon \ln (\rho+\epsilon)] d x \\
& =-\int_{B_{R}} u \cdot \nabla \rho d x-\int_{B_{R}} \operatorname{div} u \cdot \epsilon \ln (\rho+\epsilon) d x .
\end{aligned}
$$

Owing to (2.6), we then have

$$
\frac{d}{d t} \int_{B_{R}}[\rho \ln (\rho+\epsilon)+\epsilon \ln (\rho+\epsilon)] d x=\int_{B_{R}} u \cdot \nabla \rho d x+\int_{B_{R}} \operatorname{div} u \cdot \epsilon \ln (\rho+\epsilon) d x .
$$

Letting $\epsilon \rightarrow 0$, and noting that $\rho \in C\left([0, T] ; H^{m}\left(\mathbb{R}^{n}\right)\right)$ for $1 \leq n \leq 2$, the function $\rho \rightarrow$ $\rho \ln \rho$ is continuous with respect to $\rho \in[0,+\infty)$ and that $|\epsilon \ln (\rho+\epsilon)| \leq C|\epsilon \ln \epsilon| \rightarrow 0$, it is easy to calculate that

$$
\int_{B_{R}} \rho \ln \rho d x=\int_{0}^{t} \int_{B_{R}} u \cdot \nabla \rho d x d \tau+\int_{B_{R}} \rho_{0} \ln \rho_{0} d x .
$$

Subsequently, multiplying the momentum equation of (1.1) by $u$ and using the equation of density, we obtain

$$
\frac{d}{2 d t} \int_{\mathbb{R}^{n}} \rho|u|^{2} d x+\mu \int_{\mathbb{R}^{n}}|\nabla u|^{2} d x+(\lambda+\mu) \int_{\mathbb{R}^{n}}|\operatorname{div} u|^{2} d x+a \int_{\mathbb{R}^{n}} u \cdot \nabla \rho d x=0 .
$$

Subtracting this identity from (2.8) gives

$$
\begin{aligned}
& \frac{1}{2} \int_{B_{R}} \rho|u|^{2} d x+a \int_{B_{R}} \rho \ln \rho d x+\mu \int_{0}^{t} \int_{B_{R}}|\nabla u|^{2} d x d \tau+(\lambda+\mu) \int_{0}^{t} \int_{B_{R}}|\operatorname{div} u|^{2} d x d \tau \\
= & \frac{1}{2} \int_{B_{R}} \rho_{0}\left|u_{0}\right|^{2} d x+a \int_{B_{R}} \rho_{0} \ln \rho_{0} d x .
\end{aligned}
$$


Step 4. Boundedness of $\int_{B_{R}} \rho u \cdot x d x$.

Noting that $\rho \ln \rho \geq-2 / e$ for any $\rho \geq 0$, we get

$$
\int_{B_{R}} \rho \ln \rho d x \geq-C R^{n} .
$$

Then by (2.9) and (2.10), we get

$$
\int_{B_{R}} \rho|u|^{2} d x \leq C .
$$

Finally, using Cauchy's inequality,

$$
\left|\int_{B_{R}} \rho u \cdot x d x\right| \leq R^{2} \int_{B_{R}} \rho d x+\int_{B_{R}} \rho|u|^{2} d x \leq C .
$$

This completes the proof.

\section{REFERENCES}

[1] Y. Cho and B.J. Jin, Blow-up of viscous heat-conducting compressible flows, J. Math. Anal. Appl., 320, 819-826, 2006.

[2] X. D. Huang, J. Li, Z. Luo, and Z.P. Xin, Global existence and blowup phenomena for smooth solutions to the two-dimensional compressible flows, preprint.

[3] X.D. Huang, J. Li, and Z.P. Xin, Global well-posedness of classical solutions with large oscillations and vacuum to the three-dimensional isentropic compressible Navier-Stokes equations, Commun. Pure Appl. Math., 65, 549-585, 2012.

[4] T. Makino, S. Ukai, and S. Kawashima, Sur la solution à support compact de l'équations d'Euler compressible, Japan J. Appl. Math., 3, 249-257, 1986.

[5] O. Rozanova, Blow-up of smooth highly decreasing at infinity solutions to the compressible Navier-Stokes equations, J. Diff. Eqs., 245, 1762-1774, 2008.

[6] T. Sideris, Formation of singularities in solutions to nonlinear hyperbolic equations, Arch. Rational Mech. Anal., 86, 369-381, 1984.

[7] Z. Tan and Y.J. Wang, Blow-up of smooth solutions to the Navier-Stokes equations of compressible viscous heat-conducting fluids, J. Aust. Math. Soc., 88, 239-246, 2010.

[8] Z.P. Xin, Blowup of smooth solutions to the compressible Navier-Stokes equation with compact density, Commun. Pure Appl. Math., 51, 229-240, 1998.

[9] Z.P. Xin and W. Yan, On blowup of classical solutions to the compressible Navier-Stokes equations, arxiv:1204.3169v1. 